\title{
Experimental and Modeling Study of the Turning Process of PA 6/Nano Calcium Carbonate Composite
}

\author{
Mehdi Haghi, ${ }^{1}$ Reza Farshbaf Zinati, ${ }^{2}$ and Mohammad Reza Razfar ${ }^{2}$ \\ ${ }^{1}$ Department of Mechanical Engineering, University of Tabriz, Tabriz, Tabriz 51666-16471, Iran \\ ${ }^{2}$ Department of Mechanical Engineering, Amirkabir University of Technology, 424 Hafez Avenue, Tehran 15875-4413, Iran \\ Correspondence should be addressed to Reza Farshbaf Zinati; reza.farshbaf.zinati@aut.ac.ir
}

Received 19 January 2013; Revised 13 May 2013; Accepted 14 May 2013

Academic Editor: Zhiping Luo

Copyright (c) 2013 Mehdi Haghi et al. This is an open access article distributed under the Creative Commons Attribution License, which permits unrestricted use, distribution, and reproduction in any medium, provided the original work is properly cited.

\begin{abstract}
Nowadays, polymeric nanocomposites have emerged as a new material class with rapidly growing use in industrial products because of good mechanical, thermal, and physical properties. Recently, the requirement of the direct machining of these materials has increased due to the production of the most of them by extrusion method in simple cross section and the increased demand for personalized products. In this work, the effect of turning parameters (cutting speed and feed) and nano calcium carbonate content on the machinability properties of polyamide 6/nano calcium carbonate composites was investigated by analysis of variance. A novel modeling approach of modified harmony search-based neural network was also utilized to create predictive models of surface roughness and total cutting force from the experimental data. The results revealed that the nano calcium carbonate content on polyamide 6 decreased the cutting forces significantly but did not have a significant effect on surface roughness. Moreover, the results for modeling total cutting forces and surface roughness showed that modified harmony search-based neural network is effective, reliable, and authoritative in modeling the turning process of polyamide 6/nano calcium carbonate composite.
\end{abstract}

\section{Introduction}

Calcium carbonate, with 85 percent share of consumption among fillers, becomes the most widely consumed filler in the polymer industry; nevertheless, most of the studies in the field of polyamide 6 (PA 6) deal with the nanofiller of clay, and only a few studies deal with nanocomposites of polyamide 6/nano calcium carbonate (PA 6/NCC). On the other hand, findings reveal that adding nanoclay particles to polymer reduces the impact strength of the produced composite and makes it brittle whereas adding NCC increases the tensile strength of the composite thereby not making it brittle [1].

NCC has been used widely as a filler for a wide variety of resins such as polyamide (PA), polyvinyl chloride (PVC), polystyrene (PS), and polypropylene (PP) [2] because of its capability to provide increased stiffness, toughness, and dimensional stability.

Substantial improvements in mechanical, thermal, and physical properties of polymer nanocomposites have widened the use of these polymers in industry. In the late 1980s, the Toyota Motor Company commercialized a timing belt cover made from nylon 6/nanoclay composites for one of its car models, demonstrating that thermoplastic nanocomposites are one of the most promising materials to use in domestic and industrial applications [3].

An increase in the desire for personalized products has led to the need for the direct machining of polymers. The ability to machine soft materials such as polymers offers distinct advantages for producing personalized soft products using direct machining, but the machining of polymers often presents challenges to engineers in terms of close tolerances and softness, which means that it behaves differently compared to conventional metal cutting [4]. Particularly, the production of complex and unusual geometries of soft polymers by machining makes it difficult for the engineers to achieve close geometrical tolerances.

Surface roughness and total cutting force are two important criteria used to evaluate the machinability of a material. Surface properties of polymeric materials are critically important in many applications and are strongly influenced by the 


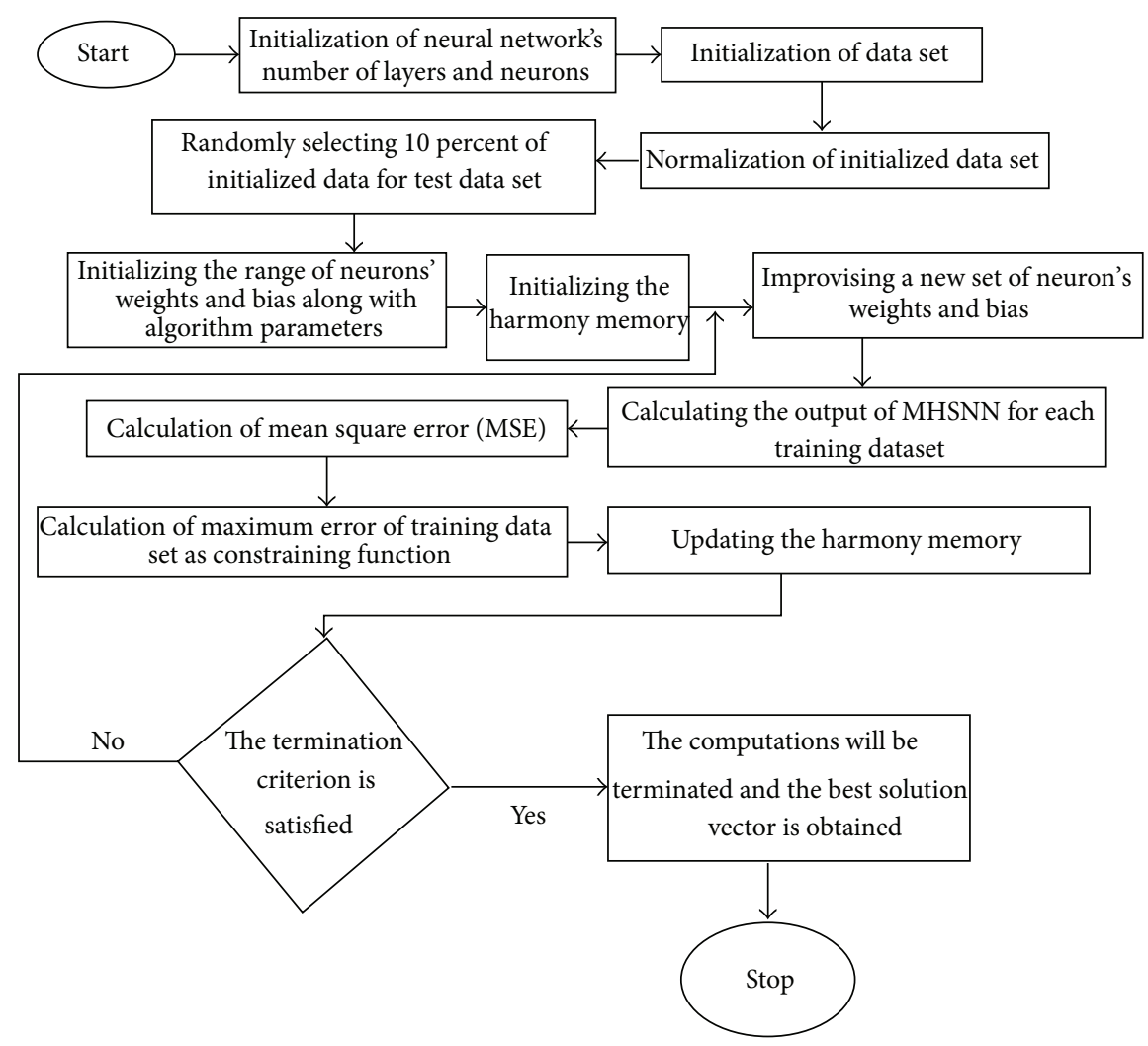

FIGURE 1: Flowchart for the MHSNN.

structure and composition of the outermost molecular layers. Appropriate surface quality is required for procedures such as coating in order to approach special surface properties like hardness, wear resistance, and friction. Cutting force analysis plays a vital role in studying the various characteristics of a machining process, like dynamic stability, positioning accuracy of the tool, and roughness of the machined surface [5].

However, a lot of information is available about the machining of metals, but not enough information exists on the machining of polymers and their composites. In recent years, a few studies have been done about the machining operations of the polymer composites. In 2006, Mata et al. [6] developed a physical cutting model for the 30 percent glass fiber reinforced PA66. In 2007, Davim and Mata [7] studied the turning process of 30 percent glass fiber reinforced and unreinforced PA 6, and Dhokia et al. [8] developed a prediction model for the surface roughness of machined polypropylene products. In 2008, Dhokia et al. [9] optimized the machining parameters for a specific surface roughness in ball-end machining of polypropylene. In 2009, Davim et al. [10] studied the machinability of 30 percent glass fiber reinforced and unreinforced PA 6 in precision turning using four distinct tool materials.

Various process-dependent factors influence the mechanism of surface roughness and cutting forces in addition to cutting parameters. Thus, it is necessary to have an effective and accurate predictive model which can be of two types: mechanistic and empirical. The mechanistic model is recognized as a functional correlation between input-output parameters appointed analytically. However, lack of accuracy and effectiveness, in addition to shortage of existing mechanistic models for surface roughness mechanism and cutting forces, has forced the researchers to rely on the empirical models. ANNs, as empirical models, are widely utilized for system modeling and optimization because it can develop nonlinear input(s)-output(s) correlation in a process without close investigation of the existing mathematical complexity in process parameters relation $[11,12]$.

In the current work, the effect of turning parameters (cutting speed and feed) and NCC content on the machinability properties of PA 6/NCC composites was studied through ANOVA and MHSNN.

\section{Modified Harmony Search-Based Neural Network (MHSNN) Algorithm}

A detailed flowchart for the MHSNN is given in Figure 1. As shown in this flowchart, the MHSNN algorithm consists of 12 steps as follows.

Step 1 (initialization of neural network's number of layers and neurons). The first step is initializing number of layers such as input layer, hidden layers, and output layer, together with each layer's number of neurons. 


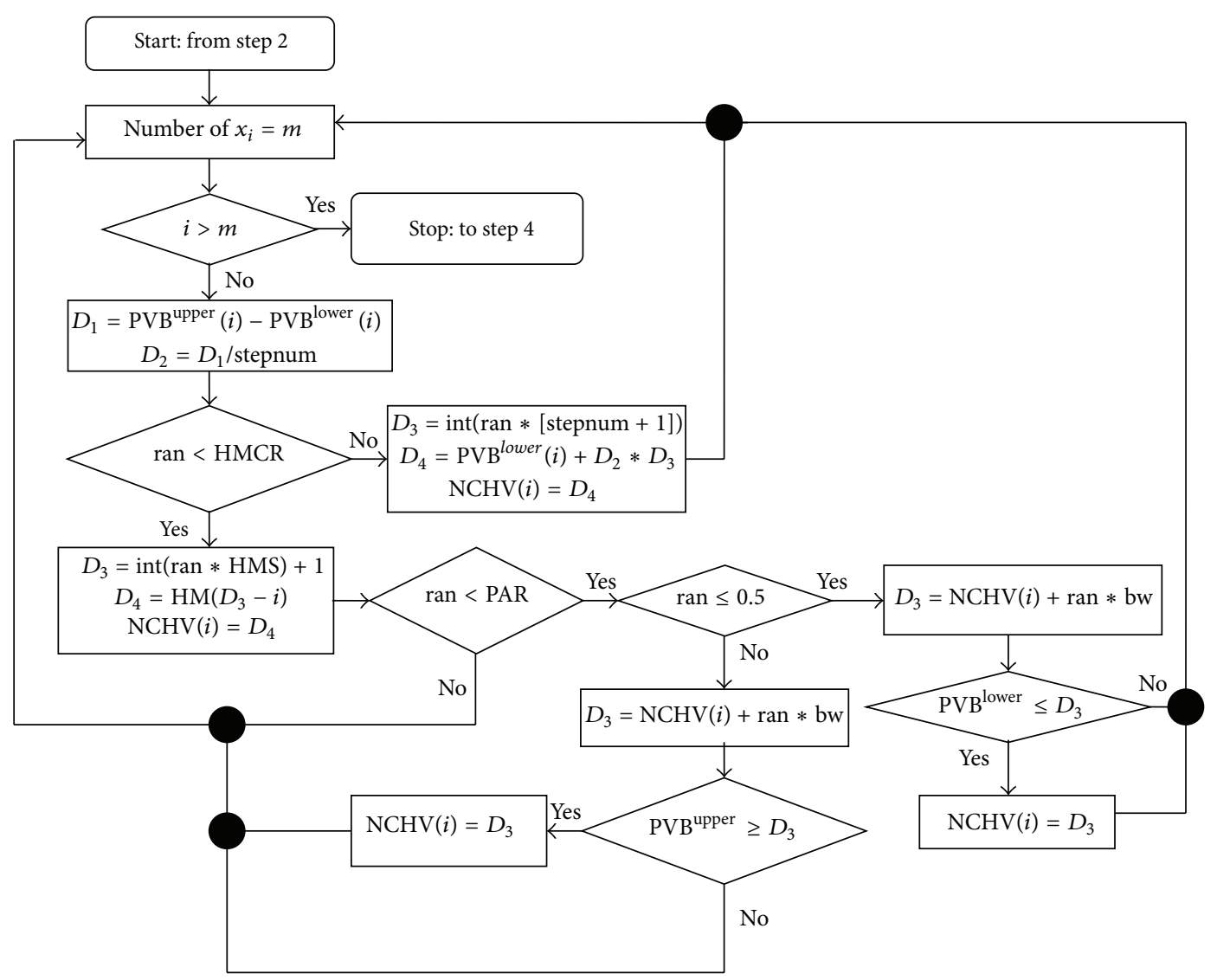

FIGURE 2: Harmony improvisation flowchart for continuous variables; (In which ran is Random numbers in the range 0-1, PVB is the possible value bound for $\mathbf{x}_{i}, \mathrm{NCHV}(\mathrm{i})$ is a new continuous harmony vector improvised in step 3 and bw is an arbitrary distance bandwidth.).

Step 2 (initialization of data set). In the second step, all data composed of input and output data sets are initialized for the algorithm.

Step 3 (normalization of initialized data set). In the third step, the input and output parameters were normalized between -1 and 1 by the following substitution:

$$
\mathbf{x}_{i}=2\left(\frac{x-x_{\min }}{x_{\max }-x_{\min }}\right)-1
$$

Step 4 (randomly selecting 10 percent of initialized data for test data set). In the forth step, 10 percent of data are selected randomly from initialized data for testing of the trained neural network. The selected data are then removed from initialized data, and the remaining data sets are assigned as training data set.

Step 5 (initializing the range of neurons' weights and bias along with algorithm parameters). The range of neurons' weights and bias together with modified harmony search (MHS) algorithm's parameters such as harmony memory size (HMS), harmony memory considering rate (HMCR), pitch adjusting rate (PAR), and number of improvisations (NI) are designated in this step.
Step 6 (initializing the harmony memory). In the sixth step, the HM matrix is filled with the number of HMS random values, in the acceptable range of each decision variable.

Step 7 (improvising a new set of neuron's weights and bias). In this step, a new set of values for all neurons' weights and bias is produced based upon considering the members existing in HM, random production of values, and pitch adjustment rule. A detailed flowchart for the continuous harmony search strategy based on the HS metaheuristic algorithm is given in Figure 2.

Step 8 (calculating the output of MHSNN for each training dataset). In Step 8, the outputs of each neuron in hidden layers and finally the output of outputlayer as the output of MHSNN are calculated according to the neuron's weights and bias values assigned by MHS algorithm.

Step 9 (calculation of mean square error (MSE)). In the ninth step, MSE for the assigned set of neuron's weights and bias is calculated according to the neural network's output and actual output of each training data set. The calculated MSE is designated as the fitness function value for the assigned set of neuron's weights and bias. 


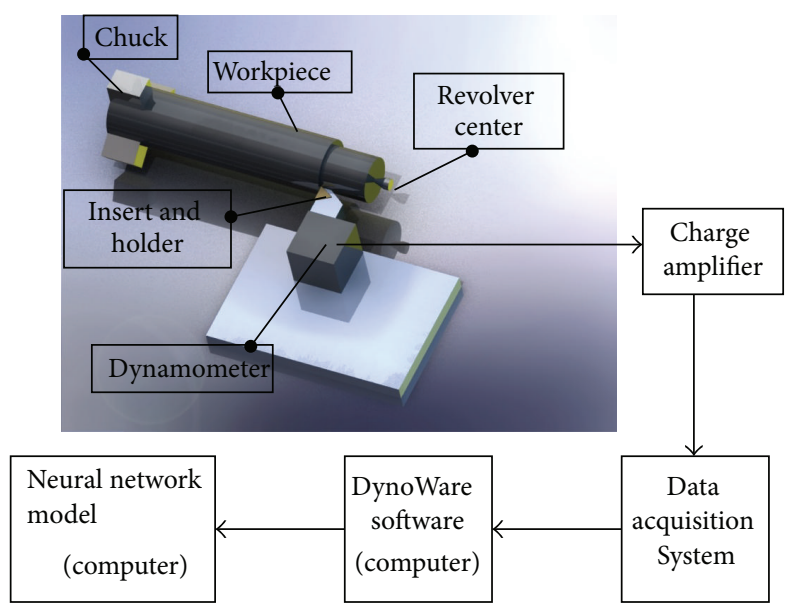

(a)

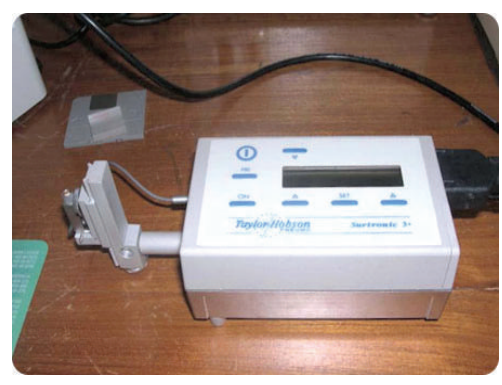

(b)

FIGURE 3: Experimental setup: (a) the schematic layout of the experimental setup and (b) the photograph of surface roughness tester.

Step 10 (calculation of maximum error of training data set as constrained function). In the tenth step, maximum error of the assigned set of neuron's weights and bias is calculated according to the neural network's output and actual output of each training data set. The calculated maximum error of training data sets is allocated as the constrained function value of assigned set of neuron's weights and bias.

Step 11 (updating the harmony memory). In step 11, the worst member of the ranked HM, $\mathbf{x}^{\#}$, is selected and compared with the new solution vector, $\mathbf{x}^{\prime}$. Note that the $\mathbf{x}^{\prime}$ does not need to be feasible. $\mathbf{x}^{\prime}$ will be replaced with $\mathbf{x}^{\#}$ only in one of the following three cases.

(1) $\mathbf{x}^{\prime}$ is feasible and $\mathbf{x}^{\#}$ is infeasible.

(2) Both $\mathbf{x}^{\prime}$ and $\mathbf{x}^{\#}$ are feasible and $F\left(\mathbf{x}^{\prime}\right)<F\left(\mathbf{x}^{\#}\right)$.

(3) Both $\mathbf{x}^{\prime}$ and $\mathbf{x}^{\#}$ are infeasible and $\mathbf{x}^{\prime}$ dominates $\mathbf{x}^{\#}$.

Step 12 (repeating Steps 6 to 10 until the termination criterion is satisfied). In Step 12, when the termination criterion is satisfied, the computations will be terminated. Otherwise, Steps 7 to 11 will be repeated. More detailed information about the MHSNN can be obtained from [11, 12].

\section{Experimental Procedure}

3.1. Material. The PA 6 with brand of "Akulon, F232-D" from DSM company was used as matrix of the composites and the NCC particles of "Socal 312" from Solvay Company with good adhesion to PA 6 used as the filler of the composites. The polypropylene modified by maleic anhydride was utilized to modify the adhesion among the polymer and nanoparticles and increase the dispersion of the nanoparticles in the polymeric matrix.

3.2. Tool and Machine Tool. The machining experiments were executed for finishing longitudinal turning on a high precision geared TN50BR lathe with $5.5 \mathrm{~kW}$ spindle motor,
TABLE 1: Levels of parameters.

\begin{tabular}{lc}
\hline Parameter & levels \\
\hline NCC $($ wt $\%)$ & $0,2.5,5,7.5,10$ \\
Cutting speed $(\mathrm{m} / \mathrm{min})$ & $21,35,43$ \\
Feed $(\mathrm{mm} / \mathrm{rev})$ & $0.1,0.15,0.2$ \\
\hline
\end{tabular}

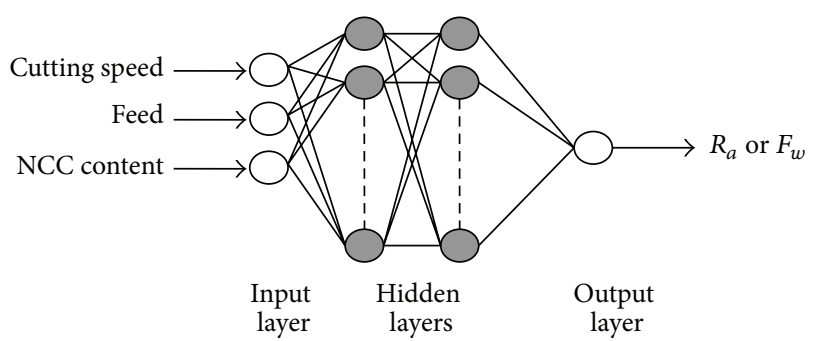

FIGURE 4: Architecture of neural network.

maximum spindle speed of $2000 \mathrm{rpm}$, and maximum feed of $6.4 \mathrm{~mm} / \mathrm{rev}$.

The used inserts were from WIDIA Company designated as ISO number of TPGN160308 and the tool holder was CTGPR-2020 K16 from MAROX Company.

Turning dynamometer 9121 model of KISTLER Company with three components as utilized to measure cutting forces. The dynamometer was fixed on tool post and the tool holder was mounted on it. During cutting operation, forces applied on the tool are converted to signals by dynamometer and amplified by charge amplifier. The amplified signals are then transferred to data acquisition card to be evaluated by DynoWare software in computer. The schematic lay out of the experimental setup is shown in Figure 3(a).

3.3. Surface Roughness Measurement. Surface roughness tool of SURTRONIC 3+ from Taylor-Hobson Company was used to measure the surface roughness $\left(R_{a}\right)$ of each experiment. To this end, three small regions on the machined surface 
TABLE 2: Comparison of neural network predictions with experimental measurement for test sets of surface roughness.

\begin{tabular}{|c|c|c|c|c|c|}
\hline \multicolumn{3}{|c|}{ Cutting condition } & \multicolumn{3}{|c|}{ Surface roughness value } \\
\hline Cutting speed $(\mathrm{m} / \mathrm{min})$ & $f(\mathrm{~mm} / \mathrm{rev})$ & NCC (wt\%) & Experiment & Predicted & Error (\%) \\
\hline 21 & 0.1 & 2.5 & 1.346 & 1.453 & 7.95 \\
\hline 43 & 0.2 & 2.5 & 5.916 & 6.121 & 3.47 \\
\hline 35 & 0.15 & 7.5 & 1.506 & 1.469 & 2.46 \\
\hline 43 & 0.15 & 10 & 2.868 & 3.008 & 4.88 \\
\hline 43 & 0.15 & 0 & 2.105 & 2.118 & 0.62 \\
\hline
\end{tabular}

TABLE 3: Comparison of neural network predictions with experimental measurement for test sets of total cutting force.

\begin{tabular}{|c|c|c|c|c|c|}
\hline \multicolumn{3}{|c|}{ Cutting condition } & \multicolumn{3}{|c|}{ Total cutting force } \\
\hline Cutting speed $(\mathrm{m} / \mathrm{min})$ & $f(\mathrm{~mm} / \mathrm{rev})$ & NCC (wt\%) & Experiment & Predicted & Error $(\%)$ \\
\hline 21 & 0.2 & 0 & 26.317 & 25.739 & 2.20 \\
\hline 43 & 0.2 & 5 & 23.696 & 23.870 & 0.73 \\
\hline 35 & 0.15 & 7.5 & 19.769 & 20.759 & 5.01 \\
\hline 21 & 0.1 & 10 & 16.961 & 17.639 & 4.00 \\
\hline 43 & 0.2 & 10 & 23.511 & 23.049 & 1.97 \\
\hline
\end{tabular}

were determined for measurements. The measurements in these regions were conducted, and the average value of three measurements was recorded as the $R_{a}$ value. The tracing velocity and the sampling length were fixed at $0.5 \mathrm{~mm} / \mathrm{s}$ and $0.8 \mathrm{~mm}$, respectively. A picture of the used tool is shown in Figure 3(b).

3.4. Design of Experiments. In this work, 45 samples based on full factorial design of the experiments employing threelevel cutting parameters and five-level NCC content are given in Table 1. The depth of cut for all experiments was $1 \mathrm{~mm}$. The levels of cutting parameters were selected considering the recommendations of tool catalogue and [13].

\section{Process Modeling through MHSNN}

In this work, two neural networks composed of one hidden layer with three inputs and one output were used to model the processes, as shown in Figure 4. The three most important input parameters are cutting speed, feed, NCC content, and the output parameters including average surface roughness and total cutting force.

The experimental data comprising 45 groups was distributed because the training subset includes 40 groups (90\%) of the data, and the randomly selected testing subset includes 5 groups (10\%) of the data. In order to find the suitable architecture of the networks, different architectures with five different combinations of training-testing data sets were studied. After 1,950 iterations of MHSNN, the 3-91, 3-7-1 architectures were found to be the most suitable ones for mean surface roughness and total cutting force, respectively. The critical parameters of harmony memory size (30), harmony memory considering rate (0.5), pitch adjusting rate (0.2), number of improvisations (1950), and distance bandwidth $\left(\left(x_{U}-x_{L}\right) / 100\right)$ were used. The comparison of MHSNN predictions with the experimental measurements for test datasets of the average surface roughness and total cutting force is illustrated in Tables 2 and 3, respectively.

\section{Results}

The analysis of variance (ANOVA) has been employed by Minitab to investigate the influence of cutting parameters and NCC content on the surface roughness and total cutting force. The mean effects of the factorial experiments on the surface roughness and total cutting force were illustrated in Figure 5. Tables 4 and 5 show the analysis of variance with the surface roughness and total cutting force, respectively. These analyses were carried out for a level of significance of $5 \%$, that is, for a level of confidence of $95 \%$.

From Table 4 it is clear that the feed factor $(P=91.64 \%)$ has statistical and physical significance on the surface roughness.

From Table 5 it is apparent that the cutting speed factor $(P=1.44 \%)$, feed factor $(P=93.89 \%)$, and NCC content factor $(P=3.26 \%)$ have statistical and physical significance on the total cutting force.

The factors and interactions present a statistical and physical significance, because $F>F_{\alpha}=5 \%$. Note that the errors associated with table ANOVA for surface roughness and total cutting force are approximately $6.59 \%$ and $1.41 \%$, respectively.

\section{Discussion}

Figure 5(a) shows the relationship between NCC content and total cutting force. As illustrated in this figure, the forces recorded when turning the PA 6/NCC composites are considerably lower compared to the pure PA 6. This is so because the NCC particles act as a lubricant in the cutting area. Therefore, the friction between the tool workpiece and chip tool decreases and causes the reduction of the total cutting force. But, the cutting area is saturated with excessive 


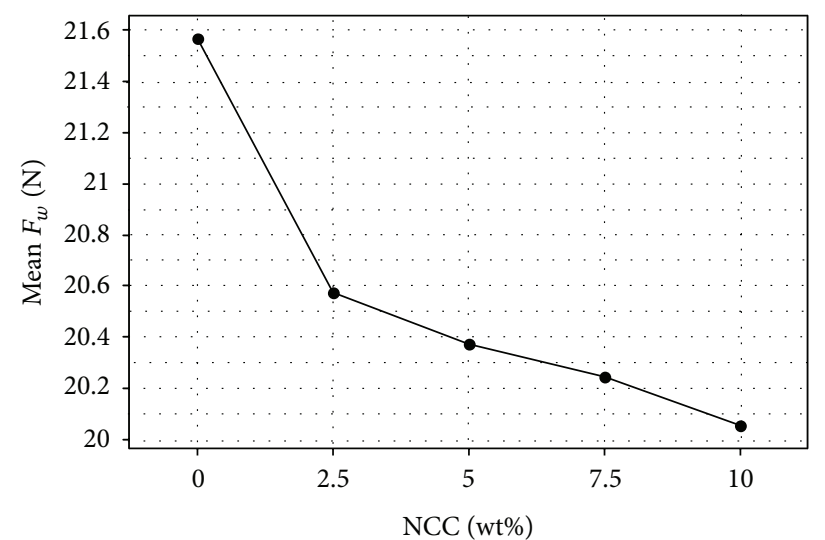

(a)

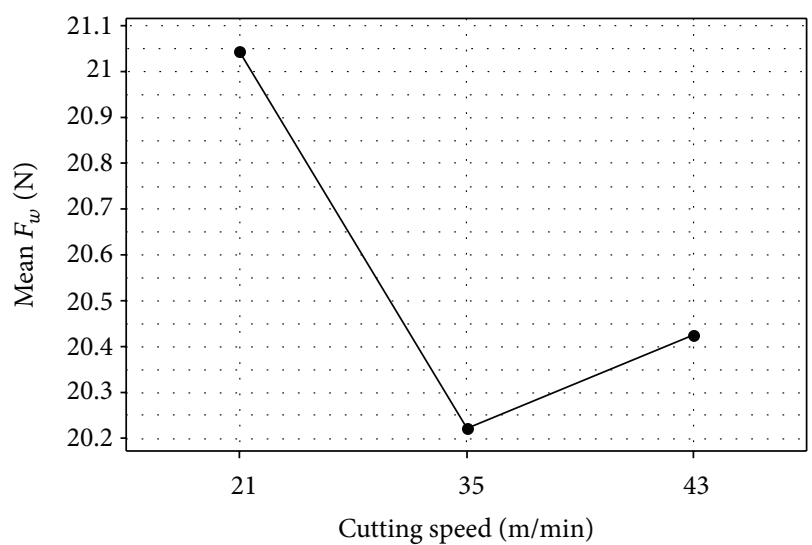

(c)

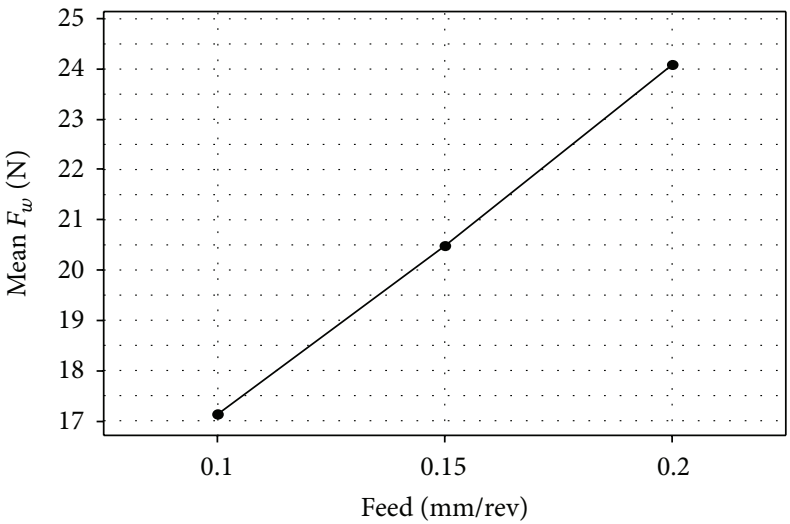

(b)

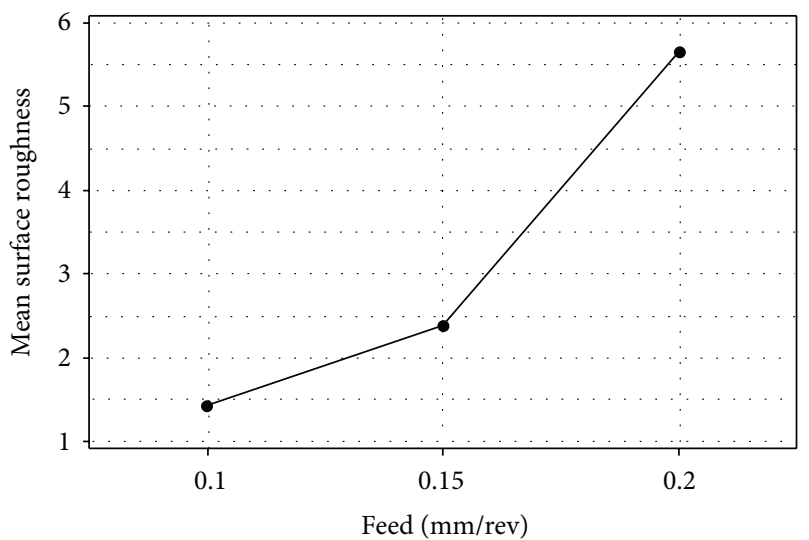

(d)

FIGURE 5: The mean effects of the factorial experiments on the surface roughness and total cutting force.

TABLE 4: Table ANOVA for the surface roughness.

\begin{tabular}{|c|c|c|c|c|c|c|}
\hline Source of variance & D.F. & Sum of square (SS) & Variance $(V)$ & $F$ & $F_{\alpha}=5 \%$ & $P(\%)$ \\
\hline$V(\mathrm{~m} / \mathrm{min})$ & 2 & 1.065 & 0.533 & 1.75 & 3.21 & 0.64 \\
\hline$f(\mathrm{~mm} / \mathrm{rev})$ & 2 & 152.417 & 76.208 & 250.68 & 3.21 & 91.64 \\
\hline NCC (wt\%) & 4 & 1.873 & 0.468 & 1.54 & 2.59 & 1.13 \\
\hline Error & 36 & 10.960 & 0.304 & - & - & 6.59 \\
\hline Total & 44 & 166.316 & - & - & - & 100 \\
\hline
\end{tabular}

increasing of the lubricant particles and the reduction of the friction becomes negligible. Thus, the slope of decreasing force is decreased by increasing the NCC content.

Figures 5(b) and 5(c) demonstrate that the $F_{w}$ increases with feed rate but decreases with the cutting speed. The first phenomenon is due to the increase of the cross-sectional area of the uncut chip which results in greater resistance in chip formation and consequently larger total cutting force. The second one is due to the thinning of the chips and the increasing of the shear angle which results in the plastic deformation of workpiece with lower strain thereby reducing the total cutting force and required power $[13,14]$.

Figure $5(\mathrm{~d})$ shows that the value of $R_{a}$ increases when the feed rate does. According to the results of ANOVA on the surface roughness data, the cutting speed and NCC content do not have any statistical and physical effect on the surface roughness.

As proved for all machining processes, two independent factors affect the final surface roughness $[13,15]$.

(a) The geometry of tool and the machining parameter of feed rate affect the final surface roughness with clear trend and result in the ideal surface roughness.

(b) The irregularities and uncontrollable factors such as vibration in the cutting operation affect the final surface roughness with unknown trend and result in the natural surface roughness.

The results illustrate that the turning of PA 6/NCC composites also follows from the general rule. 
TABLE 5: Table ANOVA for the total cutting force.

\begin{tabular}{|c|c|c|c|c|c|c|}
\hline Source of variance & D.F. & Sum of square (SS) & Variance $(V)$ & $F$ & $F_{\alpha}=5 \%$ & $P(\%)$ \\
\hline$V(\mathrm{~m} / \mathrm{min})$ & 2 & 5.561 & 2.781 & 19.01 & 3.21 & 1.44 \\
\hline$f(\mathrm{~mm} / \mathrm{rev})$ & 2 & 363.828 & 181.914 & 1204.72 & 3.21 & 93.89 \\
\hline NCC (wt\%) & 4 & 12.657 & 3.164 & 20.95 & 2.59 & 3.26 \\
\hline Error & 36 & 5.443 & 0.151 & - & - & 1.41 \\
\hline Total & 44 & 387.490 & - & - & - & 100 \\
\hline
\end{tabular}

\section{Conclusion}

This research studied the effect of NCC content on the machinability of PA 6/NCC composite and modeling its machinability parameters using MHSNN. Total cutting force and surface roughness were investigated through turning process of PA 6/NCC composite and compared with that of the pure PA 6.

The turning of PA 6/NCC composite proved to require less total cutting force than pure PA 6 . This means that the NCC particles act as a lubricant to decrease the total cutting force. The turning of PA 6/NCC composites with different NCC content and pure PA 6 proved that the NCC is inefficient in terms of surface roughness. On the other hand, the effect of cutting parameters such as cutting speed and feed on the total cutting force and surface roughness in the turning of PA 6/nano $\mathrm{CaCO}_{3}$ composites is similar to that in the turning of metals.

Modeling the mechanism of surface roughness formation and total cutting force were done by using MHSNN. The low errors in the predictions of neural networks illustrate the reliability and authority of MHSNN in modeling of PA 6/NCC composite's turning. Therefore, the obtained MHSNN models for total cutting force and surface roughness can be used to optimize the turning process. Thus, to use the proposed procedure (prediction of surface roughness and total cutting force using MHSNN for obtaining an optimal surface finish or total cutting force) similar experiments must be done, the neural network must be trained to predict the surface roughness or total cutting force in the given conditions, and the predictive model must be optimized by an appropriate optimization algorithm such as harmony search algorithm.

\section{References}

[1] S. N. Bhattacharya, M. R. Kamal, and R. K. Gupta, Polymeric Nanocomposites: Theory and Practice, Hanser Gardner, 2008.

[2] S. C. Tjong, Y. Z. Meng, and Y. Xu, "Structure and properties of polyamide-6/vermiculite nanocomposites prepared by direct melt compounding," Journal of Polymer Science B, vol. 40, no. 24, pp. 2860-2870, 2002.

[3] M. Alexandre and P. Dubois, "Polymer-layered silicate nanocomposites: preparation, properties and uses of a new class of materials," Materials Science and Engineering, vol. 28, no. 1, pp. 1-63, 2000.

[4] F. Z. Fang and Y. C. Liu, "On minimum exit-burr in micro cutting," Journal of Micromechanics and Microengineering, vol. 4, pp. 984-988, 2004.
[5] W. Wang, S. H. Kweon, and S. H. Yang, "A study on roughness of the micro-end-milled surface produced by a miniatured machine tool," Journal of Materials Processing Technology, vol. 162-163, pp. 702-708, 2005.

[6] F. Mata, P. Reis, and J. P. Davim, "Physical cutting model of polyamide composites (PA66 GF30)," Materials Science Forum, vol. 514-516, no. 1, pp. 643-647, 2006.

[7] J. P. Davim and F. Mata, "A comparative evaluation of the turning of reinforced and unreinforced polyamide," International Journal of Advanced Manufacturing Technology, vol. 33, pp. 911914, 2007.

[8] V. G. Dhokia, S. Kumar, P. Vichare, S. T. Newman, and R. D. Allen, "Surface roughness prediction model for CNC machining of polypropylene," Proceedings of the Institution of Mechanical Engineers B, vol. 222, no. 2, pp. 137-153, 2008.

[9] V. G. Dhokia, S. Kumar, P. Vichare, and S. T. Newman, "An intelligent approach for the prediction of surface roughness in ball-end machining of polypropylene," Robotics and ComputerIntegrated Manufacturing, vol. 24, no. 6, pp. 835-842, 2008.

[10] J. P. Davim, L. R. Silva, A. Festas, and A. M. Abrão, "Machinability study on precision turning of PA66 polyamide with and without glass fiber reinforcing," Materials and Design, vol. 30, no. 2, pp. 228-234, 2009.

[11] M. R. Razfar, R. Farshbaf Zinati, and M. Haghshenas, "Optimum surface roughness prediction in face milling by using neural network and harmony search algorithm," International Journal of Advanced Manufacturing Technology, vol. 52, no. 5-8, pp. 487-495, 2011.

[12] R. F. Zinati and M. R. Razfar, "Constrained optimum surface roughness prediction in turning of X20Cr13 by coupling novel modified harmony search-based neural network and modified harmony search algorithm," The International Journal of Advanced Manufacturing Technology, vol. 58, no. 1-4, pp. 93107, 2012.

[13] M. Farahnakian, M. R. Razfar, M. Moghri, and M. Asadnia, "The selection of milling parameters by the PSO-based neural network modeling method," The International Journal of Advanced Manufacturing Technology, vol. 57, no. 1-4, pp. 49-60, 2011.

[14] E. J. A. Armarego and R. H. Brown, The Machining of Metals, Prentice-Hall, Upper Saddle River, NJ, USA, 1967.

[15] M. P. Groover, Fundamentals of Modern Manufacturing, Materials, Processes, and Systems, John Wiley \& Sons, New York, Ny, USA, 2007. 

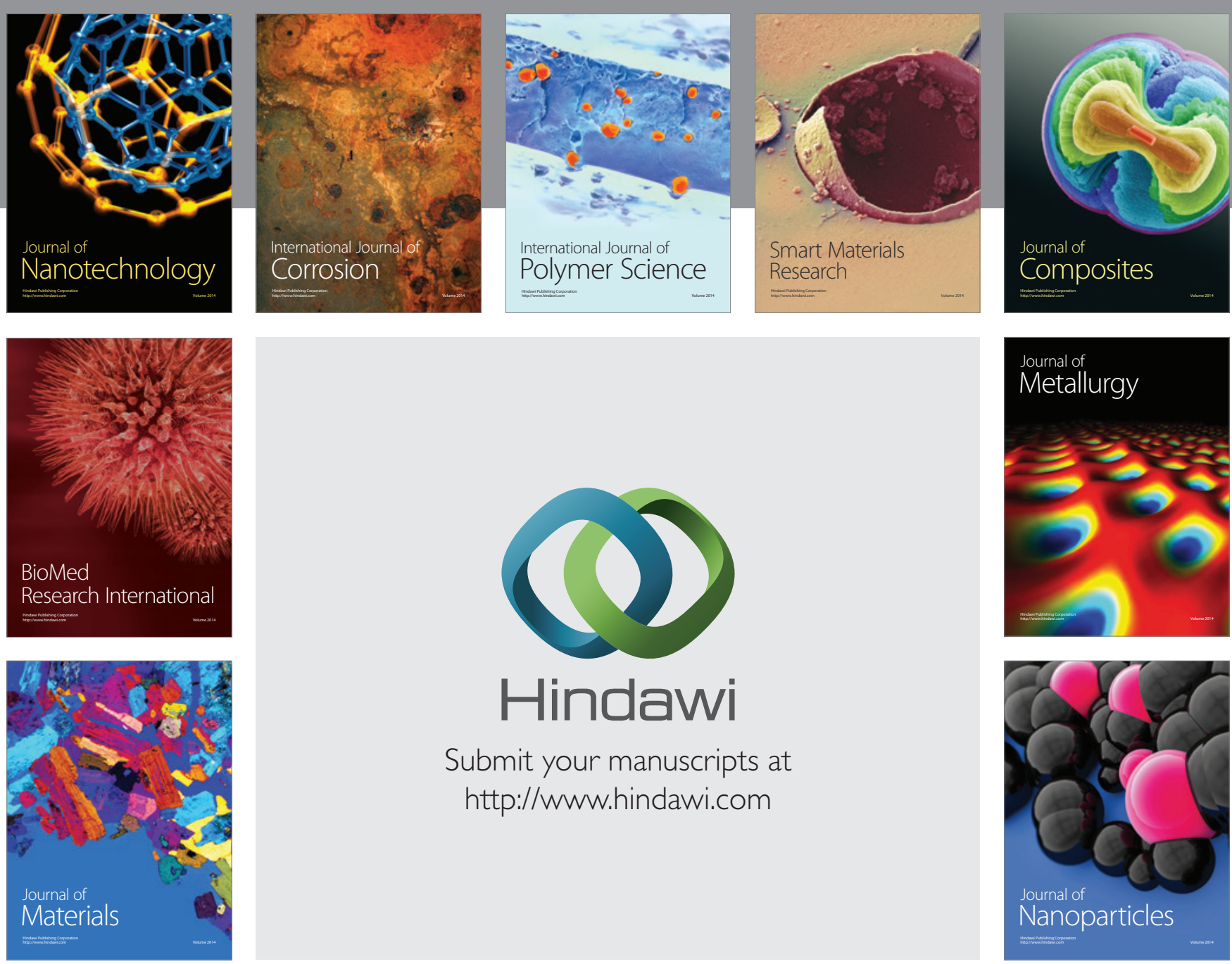

Submit your manuscripts at http://www.hindawi.com
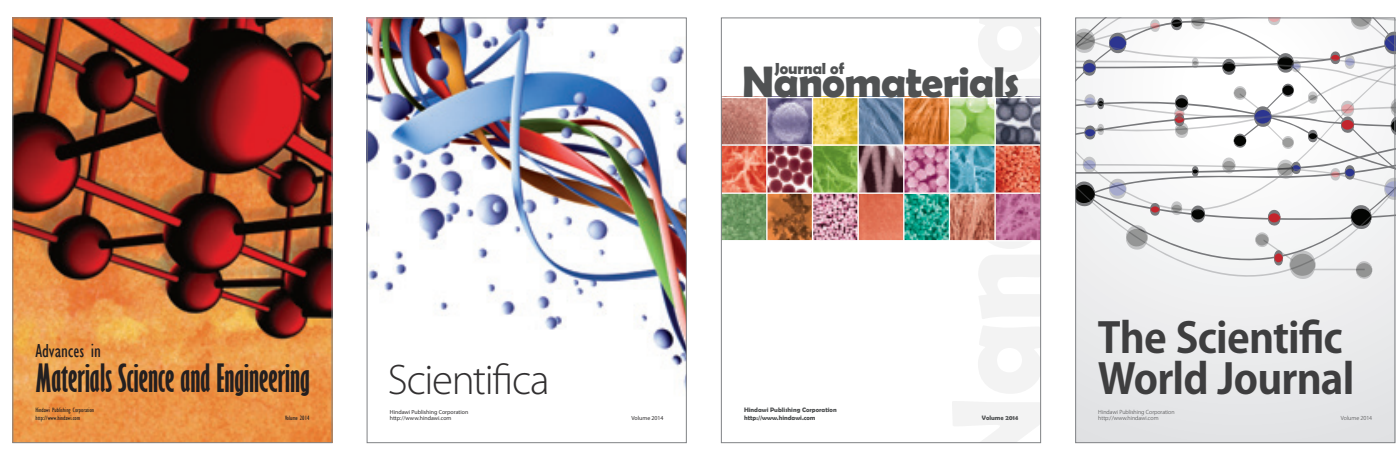

\section{The Scientific World Journal}
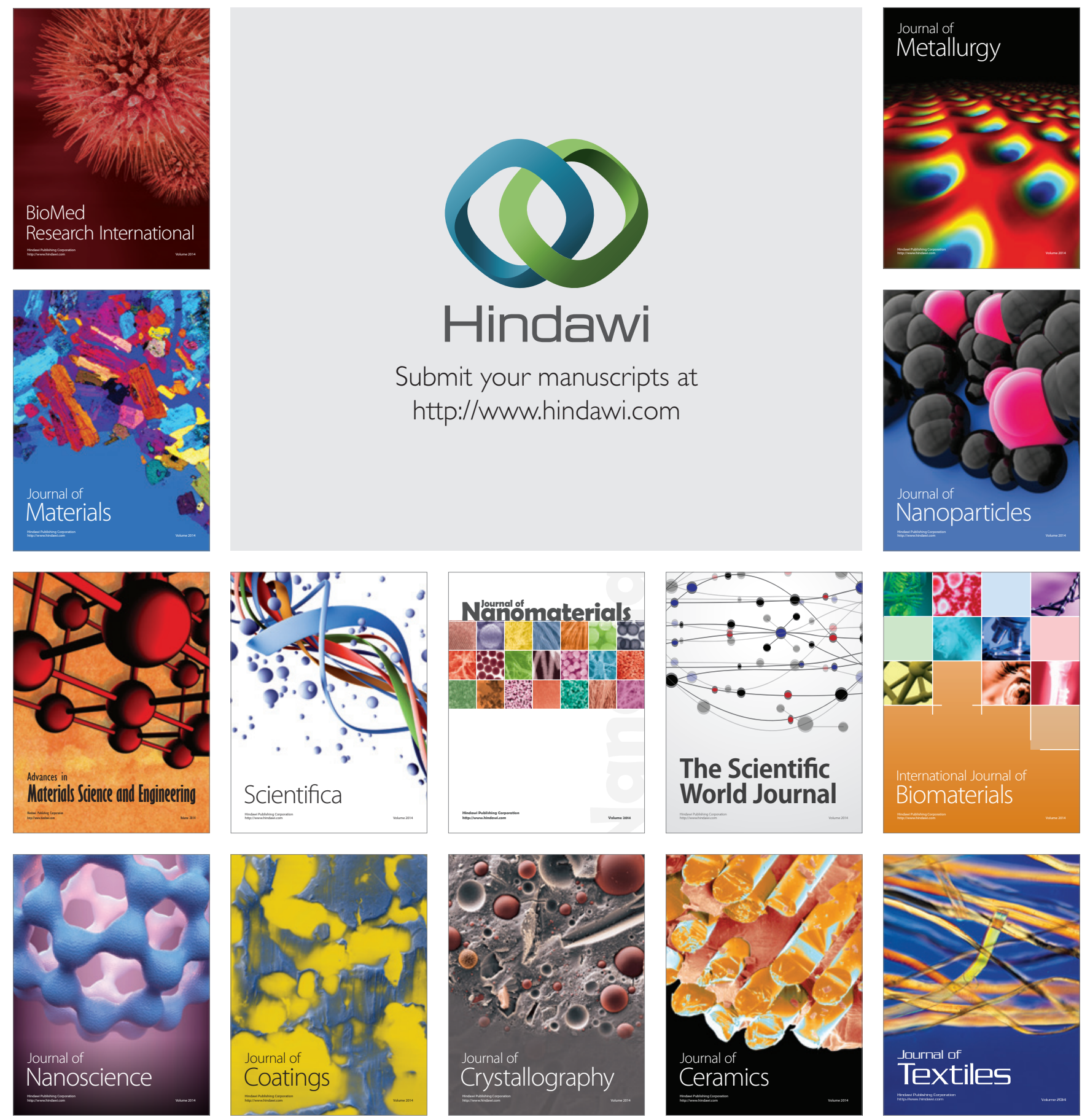